\title{
An observational study on risk of secondary cancers in chronic myeloid leukemia patients in the TKI era in the United States
}

\author{
Vivek Kumar ${ }^{\text {Corresp.. }}{ }^{1}$, Mohit Garg ${ }^{2}$, Neha Chaudhary ${ }^{3}$, Abhinav Binod Chandra ${ }^{4}$ \\ ${ }^{1}$ Department of General Internal Medicine, Brigham and Women's Hospital, Boston, Massachusetts, United States \\ 2 Department of Anaesthesia, Maimonides Medical Center, New York, United States \\ 3 Department of Pediatrics, Maimonides Medical Center, New York, United States \\ 4 Department of Hematology and Oncology, Yuma Regional Medical Center Cancer Center, Yuma, Arizona, United States \\ Corresponding Author: Vivek Kumar \\ Email address: vkumar9@bwh.harvard.edu
}

Introduction: The treatment with tyrosine kinase inhibitors (TKIs) has drastically improved the outcome of chronic myeloid leukemia (CML) patients. This study was conducted to examine the risk of secondary cancers in the CML patients who were diagnosed and treated in the TKI era in the United States. Methods: The Surveillance epidemiology and end results (SEER) database was used to identify CML patients who were diagnosed and received treatment during Jan 2002 - Dec 2014. Standardized incidence ratios (SIRs) and absolute excess risks (AER) were calculated.Results: Overall, 511 secondary cancers (excluding acute leukemia) developed in 9,200 CML patients followed for 38,433 personyears. The risk of developing secondary cancers in the CML patients was $30 \%$ higher than the age, sex and race matched standard population (SIR 1.30; 95\% Cl: 1.2-1.40; $\mathrm{p}=<0.001$ ). The SIRs for CLL (SIR 3.4, 95\% Cl: 2-5.5; $\mathrm{p}=<0.001$ ), thyroid (SIR 2.2, 95\% Cl: 1.2-3.5; $p=0.001$ ), small intestine (SIR 3.1, 95\% Cl: 1.1-6; $p=0.004$ ), gingiva (SIR 3.7, 95\% Cl: $1.2-9 ; p=0.002$ ), stomach (SIR 2.1, 95\% Cl: 1.2-3.5; $p=0.005$ ), lung (SIR 1.4, 95\% Cl: $1.1-1.7 ; p=0.006)$ and prostate (SIR $1.3,95 \% \mathrm{Cl}: 1.1-1.6 ; p=0.026)$ cancer among CML patients were significantly higher than the general population. The risk of secondary cancers was higher irrespective of age and it was highest in the period 2-12 months after the diagnosis of CML. The risk of secondary cancers in women was similar to that of the general population.Conclusion: CML patients diagnosed and treated in the TKI era in the United States are at an increased risk of developing a second malignancy. The increased risk of secondary cancers in the early period after CML diagnosis suggests that the risk of secondary cancers may be increased due to the factors other than TKIs treatment. 
2 An observational study on risk of secondary cancers in chronic myelogenous leukemia 3 patients in the TKI era in the United States.

4 Kumar, Vivek ${ }^{1}$; Garg, Mohit ${ }^{2}$; Chaudhary, Neha ${ }^{3}$; Chandra, Abhinav Binod ${ }^{4}$.

1. Department of General Internal Medicine; Brigham and Women's Hospital Boston Massachusetts USA

7

Words: Abstract: 291

Manuscript: 4166

2. Department of Anesthesia; Maimonides Medical Center New York USA

3. Department of Pediatrics; Maimonides Medical Center New York USA

4. Division of Hematology and Oncology; Yuma Regional Medical Center Cancer Center Yuma Arizona USA.

7 Corresponding Author

Abhinav Binod Chandra MD MS

Director Hematology and Oncology

Yuma Regional Medical Center Cancer Center Yuma AZ

abhinavbck@hotmail.com 
24

25

26

27

\section{Abstract}

Introduction: The treatment with tyrosine kinase inhibitors (TKIs) has drastically improved the outcome of chronic myeloid leukemia (CML) patients. This study was conducted to examine the risk of secondary cancers in the CML patients who were diagnosed and treated in the TKI era in the United States.

Methods: The Surveillance epidemiology and end results (SEER) database was used to identify CML patients who were diagnosed and received treatment during Jan 2002 - Dec 2014. Standardized incidence ratios (SIRs) and absolute excess risks (AER) were calculated.

Results: Overall, 511 secondary cancers (excluding acute leukemia) developed in 9,200 CML patients followed for 38,433 person-years. The risk of developing secondary cancers in the CML patients was $30 \%$ higher than the age, sex and race matched standard population (SIR 1.30; 95\% CI: $1.2-1.40 ; p=<0.001$ ). The SIRs for CLL (SIR 3.4, 95\% CI: 2-5.5; $p=<0.001$ ), thyroid (SIR 2.2, 95\% CI: 1.2-3.5; $p=0.001$ ), small intestine (SIR 3.1, 95\% CI: 1.1-6; $p=0.004$ ), gingiva (SIR 3.7, 95\% CI: 1.2-9; $p=0.002$ ), stomach (SIR 2.1, 95\% CI: 1.2-3.5; $p=0.005$ ), lung (SIR 1.4, 95\% CI: $1.1-1.7 ; p=0.006$ ) and prostate (SIR 1.3, 95\% CI: $1.1-1.6 ; p=0.026$ ) cancer among CML patients were significantly higher than the general population. The risk of secondary cancers was higher irrespective of age and it was highest in the period 2-12 months after the diagnosis of CML. The risk of secondary cancers in women was similar to that of the general population

Conclusion: CML patients diagnosed and treated in the TKI era in the Unites States are at an increased risk of developing a second malignancy. The increased risk of secondary cancers in the early period after CML diagnosis suggests that the risk of secondary cancers may be increased due to the factors other than the TKIs treatment. 


\section{INTRODUCTION}

47 The outcome of chronic myelogenous leukemia (CML) patients is drastically changed by tyrosine

48 kinase inhibitors (TKIs). The long term survival of CML patients who have achieved complete

49 cytogenetic remission is now similar to the general population (Gambacorti-Passerini et al. 2011).

50 The increased survival of these patients require better understanding of long term adverse effects

51 of TKIs particularly development of de novo malignancies.

52 The carcinogenic potential of imatinib was first reported in a 2-year carcinogenicity study on rats

53 in which a dose depended risk due to imatinib was observed (Fda 2018). The no observed effect

54 level (NOEL) was $15 \mathrm{mg} / \mathrm{kg} /$ day. At dose of $30 \mathrm{mg} / \mathrm{kg} /$ day onwards (approximately 0.5 or 0.3

55 times the human daily exposure at $400 \mathrm{mg} /$ day or $800 \mathrm{mg} /$ day, respectively), papilloma/carcinoma

56 of the preputial/clitoral gland were noted. At dose of $60 \mathrm{mg} / \mathrm{kg} / \mathrm{day}(\sim 1.7$ or 1 times the human

57 daily exposure at $400 \mathrm{mg} /$ day or $800 \mathrm{mg} /$ day, respectively), the renal adenoma/carcinoma, the

58 urinary bladder and urethral papilloma, the small intestine adenocarcinomas, the parathyroid

59 glands adenomas, the benign and malignant medullary tumors of the adrenal glands and the non-

60 glandular stomach papilloma/carcinomas were noted. However the relevance of these findings for

61 humans are not yet clarified, despite many years of use of TKIs (Fda 2018).

62 Many studies in the pre-TKI period reported increased risk of secondary cancers (SCs) in the CML

63 patients as compared to the general population (Frederiksen et al. 2011; Rebora et al. 2010). The

64 data on risk of SCs in the CML patients in the post TKI period are inconclusive. Roy et al. reported

65 four times higher risk of prostate cancer in a study on 1096 imatinib treated CML patients who

66 were previously treated with interferon (Roy et al. 2005). In response to this, Pilot et al. reviewed

67 Novartis registry of CML patients and concluded that the incidence of prostate cancer in imatinib

68 treated patients was not higher than general population (SIR 0.87, 95\% CI: 0.69-1.08) (Roy et al. 
69 2005). Several other studies conducted in the post TKI era reported contrary data with increased 70 risk (Gunnarsson et al. 2015), similar risk (Gugliotta et al. 2017; Miranda et al. 2016) or lower risk 71 (Verma et al. 2011) than the general population. Also there was significant heterogeneity in the 72 type of SCs reported in these studies. The higher incidence of gastrointestinal (GI), nose and throat, 73 melanoma, kidney, endocrine and non-Hodgkin's lymphoma (NHL) has been reported 74 (Gunnarsson et al. 2015; Miranda et al. 2016; Verma et al. 2011). Previous studies based on SEER 75 database analyzed data in the pre-TKI era (till 2002) and reported 16\% higher risk of SCs in CML 76 survivors while another study compared pre and post TKI treatment risks and reported 77 approximately 50\% higher risk of developing SCs in CML patients diagnosed during 2002 to 2009 as compared to the general population in the United States (U.S.) (Brenner et al. 2009; Shah \&

79 Ghimire 2014). However the actual treatment status of these patients was not disclosed in SEER database at that time. The SEER database has released customized database in April 2017 with

81 information on chemotherapy (2017b). However, it did not specify the type of treatment individual 82 patient received. Although it can be safely assumed that most of the patients with CML who were 83 diagnosed in the post TKI era, received TKIs as they are the treatment of choice for CML since 84 FDA approval in May 2001. Currently other therapies are restricted to a small number of patients 85 who are resistant to second generation TKIs or harbor TKIs resistant mutations like T3151 (Jain 86 \& van Besien 2011).

87 This study aimed to analyze the risk of SCs among patients with CML in the TKI era in the U. S.. 
88

89

90

91

92

93

94

95

96

97

98

99

100

101

102

103

104

105

106

107

108

109

110

95

\section{MATERIALS AND METHODS}

\section{The SEER Database}

The SEER program is a population based registry which is maintained by the National Cancer Institute and covers approximately $28 \%$ of the U.S. population (2017a). It publishes data on patient demographics, cancer trends, SCs, outcome, and follow-up. We analyzed data from the SEER-18 (2000-2014) database, released in April 2017.

4

96

\section{Study Population}

Patients >20 years old who were diagnosed of CML between Jan 2002 and Dec 2014, were identified using SEER*Stat, version 8.3.4 multiple primary-standardized incidence ratio (MP-SIR) session. The customized dataset contains information on chemotherapy as 'yes' or 'no/unknown' variables. The chemotherapy has been charted as 'yes' in the SEER if patient records confirmed treatment. As per the SEER* Rx Interactive Antineoplastic Drug Database chemotherapy has been recorded 'yes' for the patients who received any of the TKIs including imatinib, ponatinib, bosutinib, dasatinib and nilotinib or any other conventional chemotherapy. For this study we only included patients who were coded as 'yes' for receiving treatment. The imatinib was approved for the treatment of CML in the US by FDA in May 2001 (2001). TKIs (Imatinib or its congeners) are the drug of choice for CML patients since their FDA approval and most of the CML patients in the US have been treated with imatinib or one of the newer TKIs. We collected data on demographics, date of diagnosis of CML and secondary cancers excluding non-melanoma skin cancers, type of SCs, vital status, cause of death, and overall survival. 


\section{Patient selection}

112 A query was run in SEER stat software to identify all the patients of age 20 years old and above

113 who were diagnosed of CML and were coded 'yes' for the chemotherapy between Jan 2002 and

114 Dec 2014. Patients were excluded if they survived for less than 2 months (to adjust for surveillance

115 bias) from the date of CML diagnosis, if CML was diagnosed on autopsy or if CML was not the

116 primary cancer. A total of 9341 patients were identified who met the eligibility criteria. Patients

117 were also excluded if they received external beam radiation therapy as the initial treatment because

118 most likely these patients underwent hematopoietic stem cell transplantation (HSCT) as described

119 in the discussion below. A total of $141(<2 \%)$ patients who developed 6 secondary cancers were

120 excluded. Finally, 9200 patients were included in the analysis. The inherent risk of development

121 of AML and ALL (blast transformation) is well known in the CML patients. The cases of acute

122 leukemia were not included in the estimation of overall SCs risk. A sensitivity analysis was

123 conducted after excluding SCs which were diagnosed during the first year after diagnosis of CML

124 to adjust for the surveillance bias.

\section{Statistical Analysis}

126 The risk of SCs in CML patients was evaluated by accumulating person-years (PYs), sex, and

127 calendar-year from 2 months after diagnosis of CML to the date of death, last follow-up,

128 diagnosis of SC, or the study end (December 31, 2014), whichever occurred first. Expected SCs

129 in the CML population were calculated based on the 2000 U.S. standard population distribution,

130 by multiplying the incidence rates specific for sex, race, 5-year attained age, and calendar-year

131 by the specific person-years at risk, followed by its summation as incorporated in SEER*stat,

132 version 8.3.4. Standardized incidence ratios (SIR) was expressed as the ratio of observed to

133 expected events. The absolute excess ratio (AER per 10,000 PYs) was estimated by subtracting 
134 the expected from the observed number of secondary cancers and dividing the difference by the 135 number of PYs at risk.

136 A Poisson distribution of observed secondary cancers was assumed for calculation of the $13795 \%$ confidence intervals (CIs) and ' $p$ ' value.

\section{Results}

A total of 9200 patients were eligible for the study. These patients were followed for an average of 4.2 years accumulating 38,433 person-years. The demographic characteristics of study patients are shown in table 1 . Briefly, $41 \%$ were females, $80 \%$ were white and $44 \%$ were of age above 60 143 years (Table 1)

Overall, 511 SCs were diagnosed during the study period. The distribution of selected SCs (where at least 5 cancers were diagnosed) with their SIRs and excess risks have been shown in the fig 1 . The risk of developing SCs in the CML patients was 30\% higher than the age, sex and race matched standard population (SIR 1.3, 95\% CI: $1.2-1.4 ; p=<0.001$ ). This aggregated to an excess of 30 cancers per 10,000 person-years. The absolute risk of developing a SCs was 1.3 percent per year $149(511 / 38,433)$ in the survivors of CML.

Of 511 cancers, $94(18 \%)$ were localized to the gastrointestinal tract, $90(18 \%)$ were in the prostate, $15177(15 \%)$ were lung cancer and $78(15 \%)$ were hematological malignancies (excluding AML and ALL). The SCs whose risks were more than three times of general population included gingiva (SIR 3.7; 95\% CI: 1.2-8.7; $p=0.002)$, CLL (SIR 3.4; 95\% CI: 2-5.5; $p<0.001$ ) and small intestine 154 (SIR 3.1; 95\% CI: 1.2-7; $p=0.004)$. The risk of thyroid (SIR 2.2; 95\% CI: $1.2-3.5 ; p=0.001)$ and 155 stomach (SIR 2.1, 95\% CI: 1.2-3.5; $\mathrm{p}=0.005$ ) cancers was doubled in the survivors of CML. The 
156 risk for developing melanoma (SIR1.5; 95\% CI: 1.1-2.2; $p=0.024)$, lung cancer (SIR 1.4; 95\% CI:

$1571.1-1.7 ; p=0.006$ ) and prostate cancer (SIR 1.3; 95\% CI: 1.1-1.6; $p=0.026$ ) also increased slightly

158 but was statistically significant (Fig 1).

159 The increased risk of SC was observed only in the men who were at $40 \%$ (SIR $1.4 ; 95 \%$ CI: 1.3 -

$1601.7 p=<0.001$ ) higher risk of developing SCs after the diagnosis of CML. This contributed to 43

161 excess cancers in men per 10,000 person-years (Table 2). On the other hand, in women, the risk

162 of SC was similar to the general population (SIR 1.1;95\% CI: $0.9-1.3 ; p=0.11$ ). Also, the

163 individual cancer risk was not different in the women compared to the general population, with

164 the exception of gastric cancer whose risk was three-times higher (SIR $3.595 \%$ CI $1.4-7.3$

$165 p=<0.001$ ), colon cancer (SIR 1.7; 95\% CI: 1.03-2.7; $p=0.018$ ) and breast cancer whose risk was

166 lower than the general population (SIR 0.6; 95\% CI: 0.4-0.9 $p=0.009$ ). (Table 2)

167 When assessed by age at diagnosis of CML, 190 (37\%) SCs were diagnosed in the patients under 168 age of 60 years while 321 (63\%) SCs were diagnosed in the patients above 60 years of age. The

169 risk of developing SCs was 50\% higher in the patients below 60 years of age and $20 \%$ higher in 170 patients above 60 years of age compared to the general population. Patients below 60 years 171 developed more CLL, skin melanoma and thyroid cancers compared to the general population 172 while elderly patients were at significantly higher risk of developing cancers of gingiva and lungs.

\section{3 (Fig 2 and 3)}

\section{Follow-up of study population}

175 The highest risk of SCs was observed in the period 2-11 months after the diagnosis of CML (SIR $1761.4 ; 95 \%$ CI: 1.1-1.8 $p=<0.001$ ) (Supplementary Table 1). The risk of developing any secondary 177 cancer remained elevated up to five years from the diagnosis of CML. However approximately 
178 only one-third patients were followed for more than five years $(3,395$ patients for 9,809 person-

179 years). The higher SIRs of CLL, colon and endocrine cancers were apparent within the first year

180 after the diagnosis of CML and remained elevated in the period 1-5 years after the diagnosis of

181 CML. Other cancers whose risks were higher during 1-5 years included prostate, cervix and lung

182 and bronchus. After five years, although the overall SIR of SCs was similar to the U.S general

183 population, the individual risks of tumors of gingiva, skin and stomach were higher. After 10 years

184 the overall risk of only hematological cancers was high due to the higher risk of NHL

185 (Supplementary Table 1).

186 A sensitivity analysis was conducted by excluding all patients who survived for less than one year 187 after diagnosis of CML to adjust for the surveillance bias. Though many cases were excluded (106 188 SCs were excluded), the overall risk of SCs did not change (Table 3).

\section{Discussion}

190

191

192

193

194

195

196

197

198

199

This large population based longitudinal analysis revealed that the CML patients who were diagnosed and treated in the TKI era were at 30 percent higher risk of developing SCs as compared to the general population. The higher risk was seen in younger as well as elderly patients but was limited only to men. The risk was higher for multiple cancers including CLL, small intestine, gingiva, thyroid, melanoma, lung and prostate cancer. The overall risk of developing SCs was higher for up to five years after the diagnosis of CML.

A previous study by Shah et al. involving CML patients diagnosed in SEER database during 19922009 compared the risk of SCs using year of diagnosis as proxy of treatment without actual treatment data (Shah \& Ghimire 2014). They concluded that the risk of SCs increased in the cohort which were diagnosed and treated in the study period corresponding to TKI era. The risk in the 
200 cohort diagnosed during 2002-2009 was estimated to be 49\% higher than the general population.

201 The present study extends the data to 2014 and included only patients who were diagnosed and

202 received treatment in the TKI period. The lower (30 percent) risk in this study is likely due to the

203 exclusion of acute leukemia from the analysis similar to previous studies (Gugliotta et al. 2017;

204 Gunnarsson et al. 2015).

205

206 The higher risk observed in our study has also been reported previously. Volgova et al reported

207 1.5 times higher risk of developing SCs among 1038 patients with CML treated during 2000-2009

208 (Voglova et al. 2011). The patients were followed for mean duration of 58 months (2-214 months)

209 after starting TKIs. However in that study risks of SCs at individual sites were similar to the general

210 population. The results from this study are also concordant with the results from another large

211 population study based upon Swedish CML-register by Gunnarson et al. They reported that CML

212 patients diagnosed during 2002-2011, were at 50\% higher risk (SIR of 1.5; 95\% CI: 1.3-2) of

213 developing SCs as compared to the general population. After a follow up of 3.7 years, 7.5\%

214 patients developed SCs (Gunnarsson et al. 2015). They reported significantly higher SIRs in older

215 patients and for cancers at certain sites like GI, nose and throat. However contrary to present study

216 the risk was higher among women as compared to men. Besides, higher risk of SCs in CML

217 patients has also been reported in several small studies (Duman et al. 2012; Helbig et al. 2015;

218 Roy et al. 2005).

219 On contrary the findings in our study are discordant to that reported by Miranda et al (Miranda et

220 al. 2016). They analyzed data from the CML IV study on 1525 patients who were followed for a

221 median of 67.5 months. The overall risk of SCs $(0.9,95 \% \mathrm{CI}$; 0.6-1.2) was not higher than the

222 general population but they reported significantly higher risk of NHL among men and women. In 
223 another study by Verma et al., 103 SCs were diagnosed in 1342 CML patients (median follow up

224 of 107 months) treated with TKIs during 1998 - 2010 (Verma et al. 2011). Overall significantly

225 fewer cancers were reported than expected for the cohort with SIR of 0.6 (95\% CI: 0.4-0.8).

226 However the number of SCs in this study could have been underestimated since only the first

227 cancer was reported. Nonetheless, the risk of certain types of secondary cancers like melanoma,

228 kidney and endocrine system was higher than the age, sex and race matched standard population.

229 The risk was lower than expected for tumors at breast, prostate and digestive tract. Similarly,

230 Gugliotta et al. reported no significant increase in the risk of SCs among CML patients enrolled in

231 imatinib trials (Gugliotta et al. 2017).

232 The higher risk of certain cancers such as CLL, small intestine, gingiva, thyroid, melanoma, lung 233 and prostate cancer as compared to general population were noted in the present study. There is 234 no consistency in the pattern of SCs reported in various studies (Gunnarsson et al. 2015; Miranda 235 et al. 2016; Roy et al. 2005; Verma et al. 2011). Higher risks of GI, hepatobiliary, adrenal and 236 hematological malignancies were reported in the previous study based on SEER database (Shah \& 237 Ghimire 2014). The authors reported no change in the risk of individual cancers in the pre and post 238 TKI periods. However in the current analysis higher risks were also noted for gingiva, thyroid, 239 lung and prostate cancers. Cancers at gingiva, thyroid and small intestine contributed little to 240 excess risk despite high SIRs due to their low rate in the background population. The etiology of 241 increased risk at selected sites in the CML patients is unclear and could be due to the higher 242 prevalence of risk factors like tobacco in these patients. Unfortunately, there is no data on risk 243 factors in the SEER database to confirm this hypothesis. Moreover the higher SIRs for gingiva and 244 thyroid cancers were based on a very small number of cases which makes its interpretation 245 difficult. The risk of hematological cancers were not higher than the general population after 
246 excluding acute leukemia but the individual risk of CLL was higher in the first year after diagnosis

247 of CML while the risk of NHL was higher after 10 years from the diagnosis of CML. Nonetheless,

248 very few patients were followed beyond ten years, more data is required to ascertain the higher

249 risk of NHL in the CML patients. The lower risk of breast cancer in the women with CML similar

250 to our study has also been reported by verma et al (SIR 0.24; 0.03-0.9) (Verma et al. 2011). The

251 reason for this interesting finding is not clear but the gonadotoxicity (and resulting ovarian failure

252 which could be protective against breast cancer) due to imatinib has not been established yet.

253 Although few case reports and preliminary data suggested premature ovarian failure among the

254 patients with CML this has not been specifically tested in large prospective studies (Christopoulos

255 et al. 2009).

256 Other interesting finding from the current study was the rare occurrence of CLL among patients

257 with CML. The coexistence of CLL and CML has been described in the literature in anecdotal

258 reports (D'Arena et al. 2012; Gargallo et al. 2005). In a case where CLL followed CML 6 years

259 after its diagnosis, the genomic studies suggested separate origins for myeloid and lymphoid clones

260 which carried mutually exclusive positive genomic markers (del17q11 (CLL) and $B C R / A B L$

$261(C M L)$, supporting the two genomic events/two diseases hypothesis (D'Arena et al. 2012). In that

262 patient, CLL responded to a second generation TKI, dasatinib which is also a treatment for CML.

263 In the present study, CLL was diagnosed among $0.17 \%$ patients. 6 of these patients were diagnosed

264 within the first year of diagnosis of CML and possibility of pre-existing CLL can't be ruled out in

265 these patients. In contrast to the case reported previously, majority of these patients were males

266 with approximately 4 times higher risk of developing CLL as compared to the U.S. general

267 population. The association of CLL with CML and male predisposition warrant further studies. 
268 The reason for higher risk of SCs in CML is not clear. Imatinib has immunosuppressive properties

269 by virtue of its inhibitory effect on differentiation of dendritic cells (DCs) from CD 34+ progenitor

270 cells (Rea et al. 2004). The resulting cells in the presence of imatinib although bear resemblance

271 to normal DCs but have lower expression of surface molecules like CD1a, CD 38, major

272 histocompatibility complex (MHC) II, thus these cells are unable to mount T-cell response. Rea et

273 al. reported that in patients on imatinib treatment, DCs differentiation rates and Th1/Th2 balance

274 remained impaired despite normalization of vascular endothelial growth factors (VEGF). Imatinib

275 also inhibits T-cells proliferation by arresting cells in G0/G1 phase (Rea et al. 2004). This may be

276 more relevant to the newer TKIs with higher immunosuppressive effects (Appel et al. 2005). Other

277 possible mechanism include interference by imatinib with the DNA repair mechanisms (Majsterek

278 et al. 2006). However the carcinogenicity of TKIs has not been proven clearly in the clinical trials.

279 The SCs arising as a result of therapy are not expected to manifest until after several years of

280 treatment as suggested in several Hodgkin's lymphoma studies where relative risk for SCs was the

281 highest 5-10 years after the diagnosis of lymphoma (Schaapveld et al. 2015). In our study the

282 maximum risk of SCs, was seen in the period soon after CML diagnosis. The risk persisted in the

283 first five years from the diagnosis of CML but only fewer patients were followed past five years

284 and long term follow up studies are required to establish the period of risk. The more likely

285 explanation for increased SCs in the period soon after the diagnosis includes factors other than

286 TKIs like increase surveillance for other cancers or genetic predisposition due to CML itself (Stein

287 2012). Unfortunately, our study was not designed to establish the causation of SCs and this

288 hypothesis requires confirmation through the clinical trials or analysis of individual-level data

289 from exclusive CML registries. Nonetheless, the increased risk in these patient mandates long term

290 active surveillance for the SCs. 
292 Other possible etiologies for increased SCs in CML may include several disease related factors.

293 BCR/ABL regulates apoptosis, proliferation and intercellular interactions. It also amplifies DNA

294 damage and promote genomic instability which may increase the genetic susceptibility to acquire

295 cancers other than CML (Pawlowska \& Blasiak 2015; Skorski 2008). The studies on population

296 based registries have reported higher risk of developing SCs contrary to the randomized trials. It

297 has been hypothesized that due to better disease control in randomized trials, the propensity to

298 develop SCs remain suppressed (Miranda et al. 2016). However, this has not been confirmed yet.

299 Chemotherapies which were used prior to the introduction of TKIs like busulphan have shown to

300 be carcinogenic (Majhail et al. 2011). Moreover patients who have undergone HSCT are also at

301 higher risk of developing SCs (Yamashita et al. 2015). Total body irradiation (TBI) in combination

302 with cyclophosphamide was the preferred regimen for conditioning prior to transplant in the past

303 (Jain \& van Besien 2011). In the more recent times non-myeloablative regimens or reduced

304 intensity conditioning (RIC) are preferred and TBI (at $200 \mathrm{cGY}$ ) with fludarabine is one of them.

305 The rate of HSCT has fallen drastically in the post TKI era. In an analysis from Europe, HSCT

306 rate dropped by $69 \%$ in the year 2007 as compared to 1999 (Gratwohl et al. 2006). Currently HSCT

307 is reserved for patients with CML after failure of second generation TKIs and among patients with

308 TKI resistant mutations like T3151 (Jain \& van Besien 2011). The studies from pre-TKI era

309 reported that SCs were the cause of deaths in up to 7\% patients with CML after 10 years of follow-

310 up (Goldman et al. 2010). There was no information on patients undergoing HSCT in the SEER

311 database. However, patients who received radiation as part of the initial treatment of CML most

312 likely underwent HSCT. Besides, radiotherapy is independently associated with increased risk of

313 SCs (Bartkowiak et al. 2012). In this study a small number of patients who received RT were thus 
314 excluded from the analysis because it was difficult to dissect the impact of radiation treatment

315 from TKIs on the SCs risks. Ideally, these patients should have been censored but this was not

316 possible due to the limitation of current dataset.

317 The strengths of this study include its large sample size. This study included patients who actually

318 received treatment in the TKI era. The information on treatment in SEER is available as 'yes' or

319 'no/unknown', and is 68\% complete as compared to SEER-Medicare data. However the specificity

320 and positive predictive values are high which means that if chemotherapy is documented 'yes',

321 patient had most likely received it (States et al. 2017). Thus this data supports the analysis on

322 adverse events like SCs. Moreover the study is based on a population-based registry and more

323 accurately reflects the risk in the community, outside the controlled settings.

324 However, the findings of this study should be interpreted with caution due to the following

325 limitations. The information on treatment in the SEER database was available as 'yes' or

326 'no/unknown'. There was no information on the type of treatment or patient adherence. However

327 it could be safely assumed that most of these patients diagnosed in the TKI era received one of the

328 TKIs. Due to the large sample size, the small number of patients who could have received

329 alternative treatment would have little effect on overall analysis. The data on HSCT was also not

330 available in the SEER database. We excluded small number of patients who received radiation

331 treatment most likely as part of conditioning agent prior to HSCT, which could have affected the

332 development of SCs. Studies have reported disparity in the reporting of TKI treatment among the

333 elderly patients in the population based registries which could have misclassified some elderly

334 patients into the non-treated group which were not included in the analysis (Hoglund et al. 2013;

335 Styles et al. 2016). Lastly, the data on the cancer risk factors like smoking and genetic

336 predisposition was not available in the SEER database. 
337 In conclusion, the risk of developing SCs in CML patients in the US who were diagnosed and

338 treated after the approval of TKIs was significantly higher than the general population. Though the

339 cause of elevated risk is not clear, the diagnosis of SCs in the early period after CML diagnosis

340 suggests the linkage to CML itself rather than TKIs. Further studies are warranted for its

341 confirmation.

Acknowledgement: This study used the SEER database. The interpretation and reporting of these

344 data are the sole responsibility of the authors. The authors acknowledge the efforts of the National

345 Cancer Institute; the Office of Research, Development and Information, CMS; Information

346 Management Services (IMS), Inc.; and the Surveillance, Epidemiology, and End Results (SEER)

347 Program tumor registries in the creation of the SEER database.

348 
359

360

361

362

363

364

365

366

367

368

369

370

371

372

373

374

375

376

377

378

379

380

381

382

383

384

385

386

387

388

389

390

391

392

393

394

395

396

397

398

399

400

401

402

403

404

405

References

2001. FDA Gives Fast Approval to Gleevec in Treatment of CML | Cancer Network | The Oncology Journal. Available at http://www.cancernetwork.com/chronic-myeloid-leukemia/fda-gives-fast-approvalgleevec-treatment-cml.

2017a. About the SEER Program - SEER. Available at https://seer.cancer.gov/about/overview.html.

2017b. Surveillance, Epidemiology, and End Results Program. Available at https://seer.cancer.gov/.

Appel S, Balabanov S, Brümmendorf TH, and Brossart P. 2005. Effects of imatinib on normal hematopoiesis and immune activation. Stem Cells 23:1082-1088.

Bartkowiak D, Humble N, Suhr P, Hagg J, Mair K, Polivka B, Schneider U, Bottke D, and Wiegel T. 2012. Second cancer after radiotherapy, 1981-2007. Radiotherapy and Oncology 105:122-126.

Brenner H, Gondos A, and Pulte D. 2009. Long-term survival in chronic myelocytic leukemia after a first primary malignancy. Leukemia research 33:1604-1608.

Christopoulos C, Dimakopoulou V, and Rotas E. 2009. Primary Ovarian Insufficiency Associated with Imatinib Therapy. http://dxdoiorg/101056/NEJMc0707841. NJ200803063581019

D'Arena G, Gemei M, Luciano L, D'Auria F, Deaglio S, Statuto T, Bianchino G, Grieco V, Mansueto G, and Guariglia R. 2012. Chronic lymphocytic leukemia after chronic myeloid leukemia in the same patient: two different genomic events and a common treatment? Journal of Clinical Oncology 30:e327-e330.

Duman BB, Paydas S, Disel U, Besen A, and Gurkan E. 2012. Secondary malignancy after imatinib therapy: eight cases and review of the literature. Leukemia \& lymphoma 53:1706-1708.

Fda. 2018. GLEEVEC (imatinib mesylate) tablets Label. Available at https://webcache.googleusercontent.com/search?q=cache:OKT9bzA8DnUJ:https://www.access data.fda.gov/drugsatfda docs/label/2008/021588s024lbl.pdf+\&cd=1\&hl=en\&ct=clnk\&gl=us.

Frederiksen H, Farkas DK, Christiansen CF, Hasselbalch HC, and Sørensen HT. 2011. Chronic myeloproliferative neoplasms and subsequent cancer risk: a Danish population-based cohort study. Blood 118:6515-6520.

Gambacorti-Passerini C, Antolini L, Mahon FX, Guilhot F, Deininger M, Fava C, Nagler A, Della Casa CM, Morra E, Abruzzese E, D'Emilio A, Stagno F, le Coutre P, Hurtado-Monroy R, Santini V, Martino B, Pane F, Piccin A, Giraldo P, Assouline S, Durosinmi MA, Leeksma O, Pogliani EM, Puttini M, Jang E, Reiffers J, Piazza R, Valsecchi MG, and Kim DW. 2011. Multicenter independent assessment of outcomes in chronic myeloid leukemia patients treated with imatinib. J Nat/ Cancer Inst 103:553561. 10.1093/jnci/djr060

10.1093/jnci/djr060. Epub 2011 Mar 21.

Gargallo P, Cacchione R, Chena C, Dupont J, Garay G, Riveros D, Larripa I, and Slavutsky I. 2005. Chronic lymphocytic leukemia developing in a patient with chronic myeloid leukemia: evidence of distinct lineage-associated genomic events. Cancer genetics and cytogenetics 161:74-77.

Goldman JM, Majhail NS, Klein JP, Wang Z, Sobocinski KA, Arora M, Horowitz MM, and Rizzo JD. 2010. Relapse and late mortality in 5-year survivors of myeloablative allogeneic hematopoietic cell transplantation for chronic myeloid leukemia in first chronic phase. Journal of Clinical Oncology 28:1888-1895.

Gratwohl A, Brand R, Apperley J, Crawley C, Ruutu T, Corradini P, Carreras E, Devergie A, Guglielmi C, and Kolb H-J. 2006. Allogeneic hematopoietic stem cell transplantation for chronic myeloid leukemia in Europe 2006: transplant activity, long-term data and current results. An analysis by the Chronic Leukemia Working Party of the European Group for Blood and Marrow Transplantation (EBMT). Haematologica 91:513-521.

PeerJ reviewing PDF | (2017:10:21289:2:0:NEW 17 Jan 2018) 
406

407

408

409

410

411

412

413

414

415

416

417

418

419

420

421

422

423

424

425

426

427

428

429

430

431

432

433

434

435

436

437

438

439

440

441

442

443

444

445

446

447

448

449

450

451

452

Gugliotta G, Castagnetti F, Breccia M, Albano F, lurlo A, Intermesoli T, Abruzzese E, Levato L, D'Adda M, and Pregno P. 2017. Incidence of second primary malignancies and related mortality in imatinibtreated chronic myeloid leukemia patients. Haematologica:haematol. 2017.169532.

Gunnarsson N, Stenke L, Höglund M, Sandin F, Björkholm M, Dreimane A, Lambe M, Markevärn B, Olsson-Strömberg $U$, and Richter J. 2015. Second malignancies following treatment of chronic myeloid leukaemia in the tyrosine kinase inhibitor era. British journal of haematology 169:683688.

Helbig G, Bober G, Seweryn M, Wichary R, Tukiendorf A, Sedlak L, Oleksy T, and Kyrcz-Krzemień S. 2015. Occurrence of secondary malignancies in chronic myeloid leukemia during therapy with imatinib mesylate-single institution experience. Mediterranean journal of hematology and infectious diseases 7.

Hoglund M, Sandin F, Hellstrom K, Bjoreman M, Bjorkholm M, Brune M, Dreimane A, Ekblom M, Lehmann S, Ljungman P, Malm C, Markevarn B, Myhr-Eriksson K, Ohm L, Olsson-Stromberg U, Sjalander A, Wadenvik H, Simonsson B, Stenke L, and Richter J. 2013. Tyrosine kinase inhibitor usage, treatment outcome, and prognostic scores in CML: report from the population-based Swedish CML registry. Blood 122:1284-1292. 10.1182/blood-2013-04-495598

10.1182/blood-2013-04-495598. Epub 2013 Jul 10.

Jain N, and van Besien K. 2011. Chronic myelogenous leukemia: role of stem cell transplant in the imatinib era. Hematology/oncology clinics of North America 25:1025-1048.

Majhail NS, Brazauskas R, Rizzo JD, Sobecks RM, Wang Z, Horowitz MM, Bolwell B, Wingard JR, and Socie G. 2011. Secondary solid cancers after allogeneic hematopoietic cell transplantation using busulfan-cyclophosphamide conditioning. Blood 117:316-322. 10.1182/blood-2010-07-294629

10.1182/blood-2010-07-294629. Epub 2010 Oct 6.

Majsterek I, Arabski M, Czechowska A, Pytel D, Morawiec Z, Morawiec-Bajda A, and Blasiak J. 2006. Imatinib (STI571) inhibits DNA repair in human leukemia oncogenic tyrosine kinase-expressing cells. Zeitschrift für Naturforschung C 61:896-902.

Miranda MB, Lauseker M, Kraus MP, Proetel U, Hanfstein B, Fabarius A, Baerlocher GM, Heim D, Hossfeld DK, Kolb HJ, Krause SW, Nerl C, Brümmendorf TH, Verbeek W, Fauser AA, Prümmer O, Neben K, Hess U, Mahlberg R, Plöger C, Flasshove M, Rendenbach B, Hofmann WK, Müller MC, Pfirrmann M, Hochhaus A, Hasford J, Hehlmann R, and Saußele S. 2016. Secondary malignancies in chronic myeloid leukemia patients after imatinib-based treatment: long-term observation in CML Study IV. Leukemia, 1255-1262.

Pawlowska E, and Blasiak J. 2015. DNA Repair-A Double-Edged Sword in the Genomic Stability of Cancer Cells-The Case of Chronic Myeloid Leukemia. International journal of molecular sciences 16:27535-27549.

Rea NB, Rousselot P, Raffoux E, Cayuela JM, Maarek O, Charron D, Degos L, Dombret H, and Toubert A. 2004. Defective blood dendritic cells in chronic myeloid leukemia correlate with high plasmatic VEGF and are not normalized by imatinib mesylate. Leukemia 18:1656-1661. doi:10.1038/sj.leu.2403474

Rebora P, Czene K, Antolini L, Passerini CG, Reilly M, and Valsecchi MG. 2010. Are chronic myeloid leukemia patients more at risk for second malignancies? A population-based study. American journal of epidemiology 172:1028-1033.

Roy L, Guilhot J, Martineau G, Larchee R, and Guilhot F. 2005. Unexpected occurrence of second malignancies in patients treated with interferon followed by imatinib mesylate for chronic myelogenous leukemia. Leukemia 19:1689-1689.

Schaapveld M, Aleman BMP, van Eggermond AM, Janus CPM, Krol ADG, van der Maazen RWM, Roesink J, Raemaekers JMM, de Boer JP, Zijlstra JM, van Imhoff GW, Petersen EJ, Poortmans PMP, Beijert 
453

454

455

456

457

458

459

460

461

462

463

464

465

466

467

468

469

470

471

472

473

474

475

476

477

478

479

480

481

482

483

484

485

486

487

M, Lybeert ML, Mulder I, Visser O, Louwman MWJ, Krul IM, Lugtenburg PJ, and van Leeuwen FE. 2015. Second Cancer Risk Up to 40 Years after Treatment for Hodgkin's Lymphoma. http://dxdoiorg/101056/NEJMoa1505949. NJ201512243732606

Shah BK, and Ghimire KB. 2014. Second primary malignancies in chronic myeloid leukemia. Indian Journal of Hematology and Blood Transfusion 30:236-240.

Skorski T. 2008. BCR/ABL, DNA damage and DNA repair: implications for new treatment concepts. Leukemia \& lymphoma 49:610-614.

States, Timothy AMN, National Cancer Institute United S, Jennifer LL, jlund@email.unc.edu, Epidemiology, Angela M, National Cancer Institute United S, Kathleen C, and National Cancer Institute U. 2017. Comparison of SEER Treatment Data With Medicare Claims. Medical Care 54. 10.1097/MLR.0000000000000073

Stein BL. 2012. Chronic myeloid leukemia and risk of second malignancy in two eras of treatment. Leukemia \& lymphoma 53:1651-1653.

Styles T, Wu M, Wilson R, Babcock F, Butterworth D, West DW, and Richardson LC. 2016. Populationbased Testing and Treatment Characteristics for Chronic Myelogenous Leukemia. J Registry Manag 41:134-142.

Verma D, Kantarjian H, Strom SS, Rios MB, Jabbour E, Quintas-Cardama A, Verstovsek S, Ravandi F, O'Brien $S$, and Cortes J. 2011. Malignancies occurring during therapy with tyrosine kinase inhibitors (TKIs) for chronic myeloid leukemia (CML) and other hematologic malignancies. Blood 118:4353-4358.

Voglova J, Muzik J, Faber E, Zackova D, Klamova H, Steinerova K, Michalovicova Z, Demitrovicova L, Cmunt E, and Novakova L. 2011. Incidence of second malignancies during treatment of chronic myeloid leukemia with tyrosine kinase inhibitors in the Czech Republic and Slovakia. Neoplasma 58:256262.

Yamashita, Takahiro Fukuda YT, Saiko K, Kinuko T, Yoshihiro I, Reiko I, Takashi T, Yoshitaka I, Takayuki O, Akihisa K, Akio O, Keiji O, Shigeo F, Sung-Won K, Ryuji T, and Takuya. 2015. Secondary Cancer after Allogeneic Hematopoietic Stem Cell Transplantation. Blood.

Fig 1. Standardized incidence ratios (SIR) and absolute excess risk (AER) of selected secondary cancers in CML patients

Fig 2. Standardized incidence ratios (SIR) and absolute excess risk (AER) of selected secondary cancers in CML patients aged 20-59 years

Fig 3. Standardized incidence ratios (SIR) and absolute excess risk (AER) of selected secondary cancers in CML patients aged 60-85+ years 
Figure 1

Standardized incidence ratios (SIR) and absolute excess risk (AER) of selected secondary cancers in CML patients

Absolute excess risk is per 10,000 individuals.

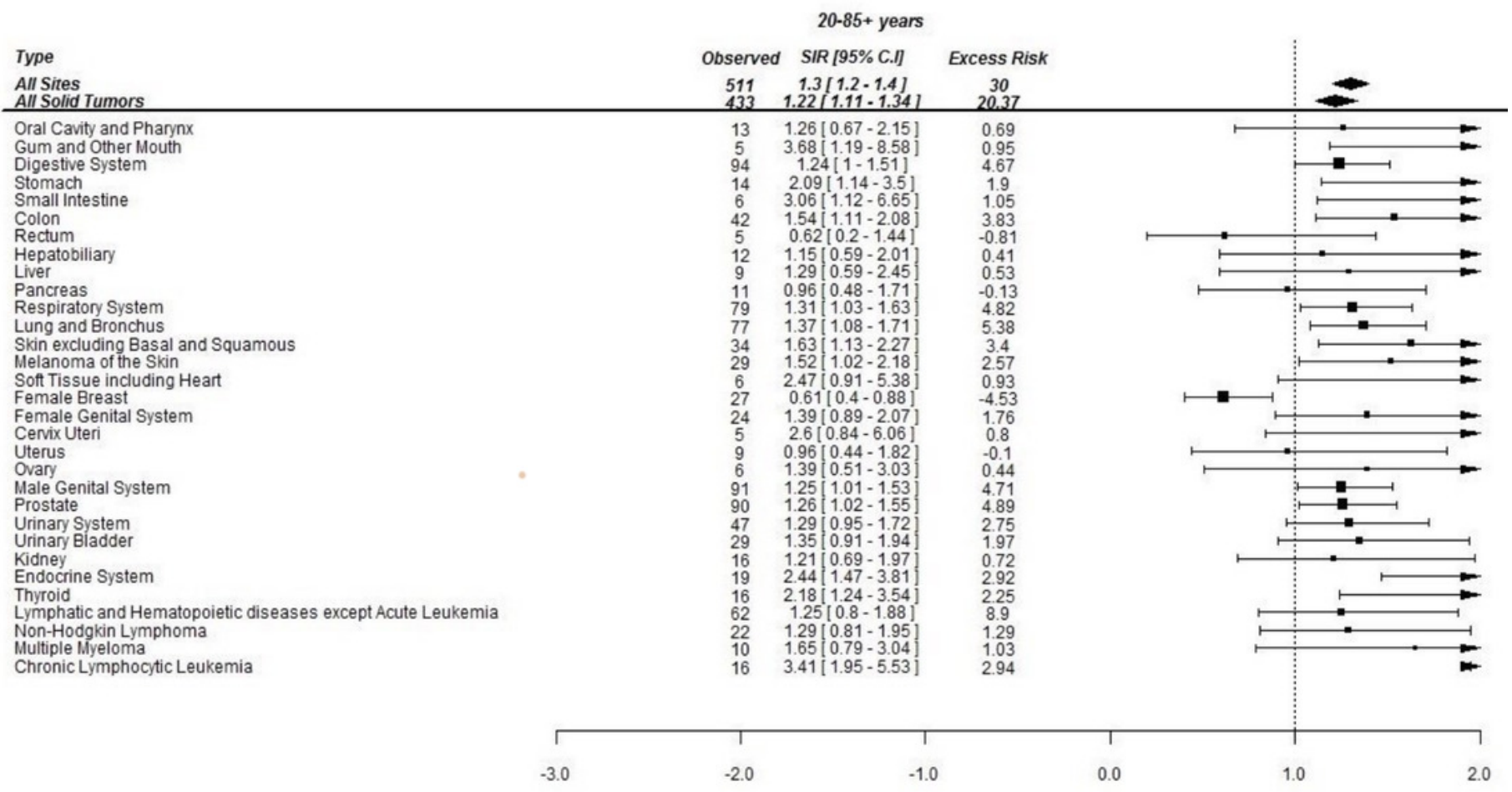


Figure 2

Standardized incidence ratios (SIR) and absolute excess risk (AER) of selected secondary cancers in CML patients aged 20-59 years

Absolute excess risk is per 10,000 individuals.

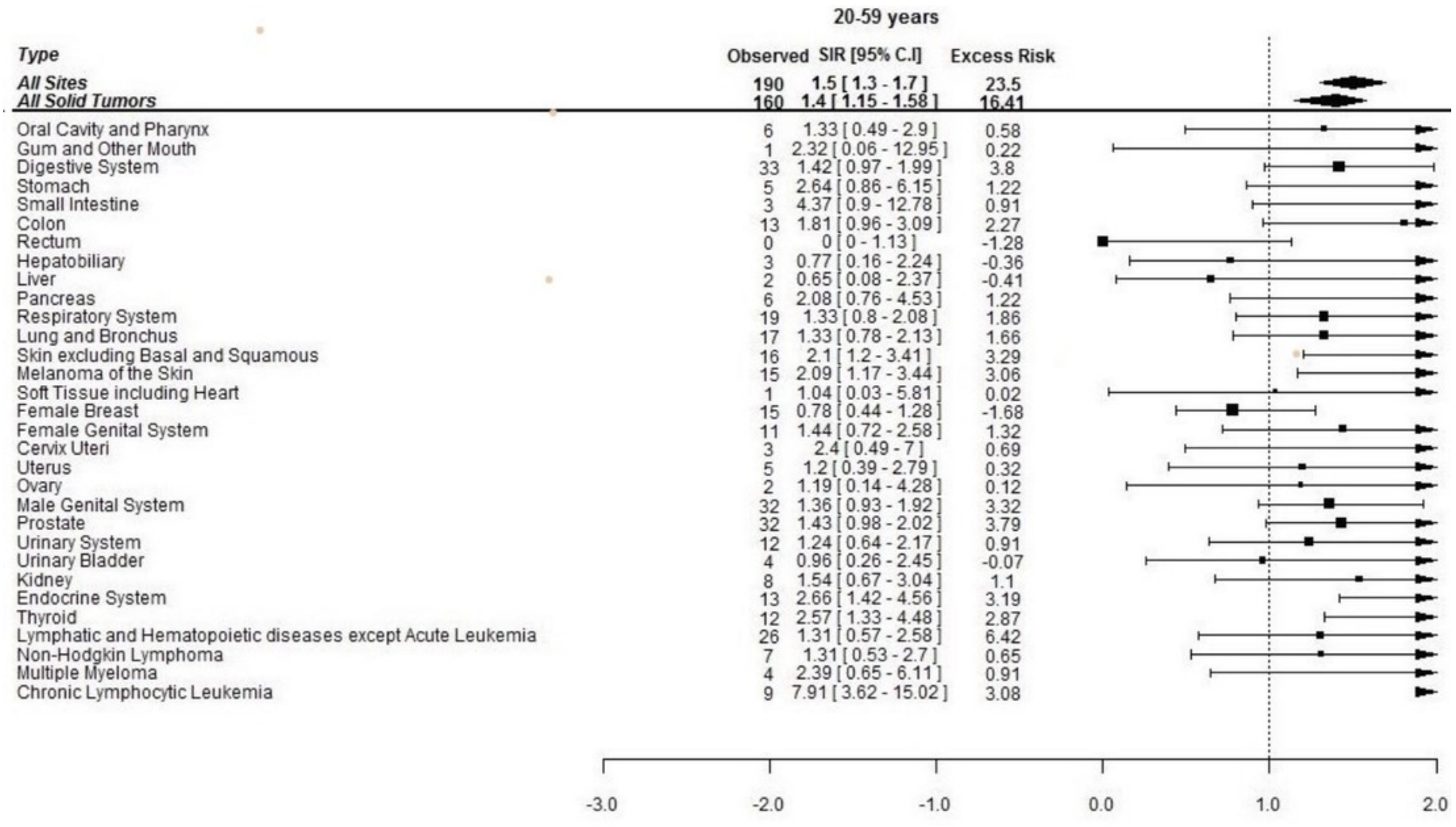


Figure 3

Standardized incidence ratios (SIR) and absolute excess risk (AER) of selected secondary cancers in CML patients aged 60-85+ years

Excess risk is per 10,000 individuals.

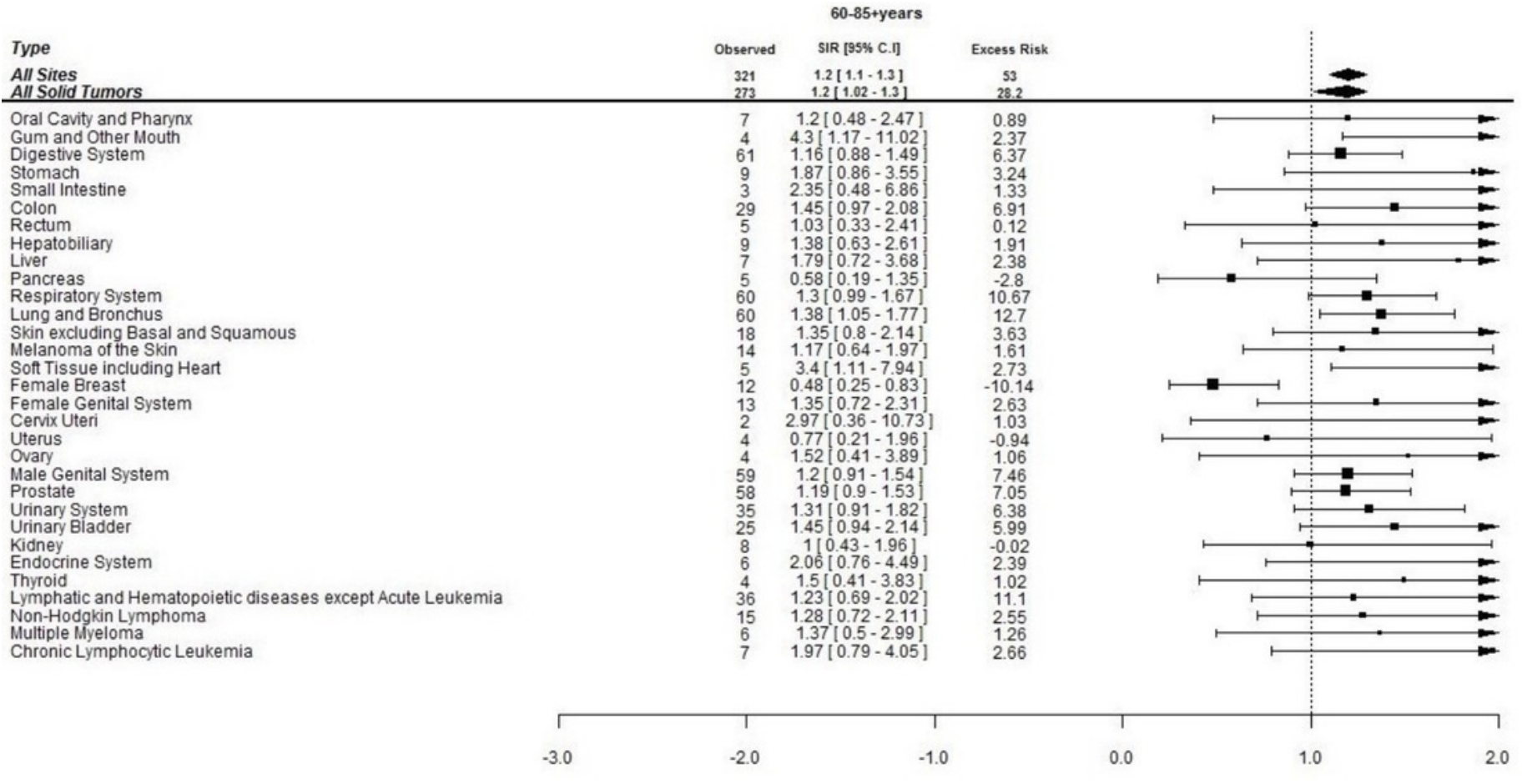


Table $\mathbf{1}$ (on next page)

Demographic characteristics of study population 
1 Table 1. Demographic characteristics of study population

\begin{tabular}{|l|l|}
\hline Demographic Characteristics & $\mathrm{N}=9,200(\mathbf{1 0 0 \%})$ \\
\hline Gender &
\end{tabular}

Male

Female

$5,420(59)$

Age (in years)

$<60$

$\geq 60$

$3,780(41)$

Ethnicity

White

African-American

American Indian/Alaska Native

Asian/Pacific Islander

Unknown

Marital Status

Married

Single

Previously married

Unknown

Geographical Location

Northern Plains

East

Pacific coast

Southwest

$5190(56)$

4010 (44)

Outcome at study cut-off

Alive

Dead

$7,338(80)$

$1,050(11)$

$55(<1)$

$625(7)$ 132(1)

$5,067(55)$

$1,756(19)$

$1,654(18)$

$723(8)$

3

4 


\section{Table 2 (on next page)}

SIR and Excess risk of Secondary Cancers among patients with CML stratified by gender

*After excluding acute leukemia NA Not applicable 
Talble 2. SIR and Excess risk of Secondary Cancers among patients with CML stratified by gender

\begin{tabular}{|c|c|c|c|c|c|c|c|c|}
\hline \multirow[b]{2}{*}{ Cancer Sites } & \multicolumn{4}{|c|}{ Male } & \multicolumn{4}{|c|}{ Female } \\
\hline & Observed & $\operatorname{SIR}(95 \% \mathrm{CI})$ & $\begin{array}{c}\text { Excess } \\
\text { Risk }\end{array}$ & $\begin{array}{c}\text { 'P' } \\
\text { value }\end{array}$ & Observed & $\operatorname{SIR}(95 \% \mathrm{CI})$ & $\begin{array}{c}\text { Excess } \\
\text { Risk }\end{array}$ & $\begin{array}{c}\text { 'P' } \\
\text { value }\end{array}$ \\
\hline All Sites * & 342 & $1.4(1.25-1.65)$ & 43.13 & $<0.001$ & 169 & $1.14(0.94-1.34)$ & 9.85 & 0.33 \\
\hline All Solid Tumors & 285 & $1.29(1.15-1.45)$ & 28.97 & $<0.001$ & 148 & $1.1(0.93-1.3)$ & 8.61 & 0.24 \\
\hline Oral Cavity and Pharynx & 11 & $1.37(0.68-2.44)$ & 1.33 & 0.29 & 2 & $0.87(0.11-3.14)$ & -0.19 & 0.84 \\
\hline Gum and Other Mouth & 4 & $4.66(1.3-11.93)$ & 1.41 & $<0.001$ & 1 & $1.99(0.05-11.11)$ & 0.31 & 0.49 \\
\hline Digestive System & 58 & $1.19(0.9-1.53)$ & 4.08 & 0.18 & 36 & $1.33(0.93-1.84)$ & 5.46 & 0.09 \\
\hline Stomach & 7 & $1.48(0.6-3.05)$ & 1.02 & 0.29 & 7 & $3.53(1.42-7.27)$ & 3.09 & $<0.001$ \\
\hline Small Intestine & 3 & $2.43(0.5-7.09)$ & 0.79 & 0.11 & 3 & $4.13(0.85-12.06)$ & 1.4 & 0.06 \\
\hline Colon & 23 & $1.42(0.9-2.14)$ & 3.08 & 0.09 & 19 & $1.71(1.03-2.67)$ & 4.87 & 0.02 \\
\hline Rectum & 5 & $0.91(0.3-2.13)$ & -0.21 & 0.83 & 0 & $0(0-1.4)$ & -1.63 & 0.8 \\
\hline Hepatobiliary System & 10 & $1.32(0.63-2.43)$ & 1.1 & 0.38 & 2 & $0.69(0.08-2.51)$ & -0.54 & 0.6 \\
\hline Liver & 7 & $1.25(0.5-2.57)$ & 0.63 & 0.55 & 2 & $1.47(0.18-5.3)$ & 0.39 & 0.58 \\
\hline Pancreas & 6 & $0.87(0.32-1.89)$ & -0.41 & 0.73 & 5 & $1.09(0.35-2.54)$ & 0.25 & 0.85 \\
\hline Respiratory System & 48 & $1.24(0.91-1.64)$ & 4.17 & 0.13 & 31 & $1.43(0.97-2.03)$ & 5.72 & 0.06 \\
\hline Lung and Bronchus & 48 & $1.36(1-1.8)$ & 5.72 & 0.03 & 29 & $1.38(0.92-1.98)$ & 4.91 & 0.08 \\
\hline Soft Tissue and Heart & 5 & $3.14(1.02-7.33)$ & 1.53 & 0.007 & 1 & $1.2(0.03-6.66)$ & 0.1 & 0.85 \\
\hline Skin (except Basal/ Squamous) & 25 & $1.68(1.09-2.48)$ & 4.55 & 0.008 & 9 & $1.5(0.68-2.84)$ & 1.84 & 0.22 \\
\hline Melanoma (Skin) & 21 & $1.54(0.95-2.36)$ & 3.33 & 0.06 & 8 & $1.46(0.63-2.87)$ & 1.55 & 0.28 \\
\hline Female Breast & NA & NA & NA & - & 27 & $0.61(0.4-0.88)$ & -10.73 & 0.009 \\
\hline Female Genital System & NA & NA & NA & - & 24 & $1.39(0.89-2.07)$ & 4.17 & 0.1 \\
\hline Cervix Uteri & NA & NA & NA & - & 5 & $2.6(0.84-6.06)$ & 1.89 & 0.08 \\
\hline Corpus and Uterus, NOS & NA & NA & NA & - & 9 & $0.96(0.44-1.82)$ & -0.25 & 0.89 \\
\hline Ovary & NA & NA & NA & - & 6 & $1.39(0.51-3.03)$ & 1.04 & 0.42 \\
\hline Male Genital System & 91 & $1.25(1.01-1.53)$ & 8.16 & 0.03 & NA & NA & NA & - \\
\hline Prostate & 90 & $1.26(1.02-1.55)$ & 8.46 & 0.02 & NA & NA & NA & - \\
\hline Urinary System & 38 & $1.33(0.94-1.83)$ & 4.26 & 0.08 & 9 & $1.14(0.52-2.17)$ & 0.69 & 0.69 \\
\hline Urinary Bladder & 26 & $1.47(0.96-2.15)$ & 3.72 & 0.06 & 3 & $0.81(0.17-2.37)$ & -0.43 & 0.72 \\
\hline
\end{tabular}




\begin{tabular}{|c|c|c|c|c|c|c|c|c|}
\hline Kidney & 11 & $1.15(0.57-2.06)$ & 0.65 & 0.64 & 5 & $1.37(0.44-3.19)$ & 0.83 & 0.48 \\
\hline Endocrine System & 10 & $3.45(1.66-6.35)$ & 3.2 & $<0.001$ & 9 & $1.84(0.84-3.49)$ & 2.53 & 0.06 \\
\hline Thyroid & 8 & 3.04 (1.31-5.99) & 2.42 & $<0.001$ & 8 & $1.7(0.73-3.34)$ & 2.02 & 0.13 \\
\hline Hematological System* & 47 & $2.2(1.1-3.3)$ & 10.82 & $<0.001$ & 19 & $1.7(0.5-3)$ & 1.2 & 0.1 \\
\hline Non-Hodgkin Lymphoma & 15 & $1.38(0.77-2.27)$ & 1.85 & 0.22 & 7 & $1.14(0.46-2.34)$ & 0.51 & 0.73 \\
\hline Myeloma & 7 & $1.77(0.71-3.64)$ & 1.37 & 0.13 & 3 & $1.44(0.3-4.21)$ & 0.57 & 0.53 \\
\hline Chronic Lymphocytic Leukemia & 13 & $3.99(2.13-6.82)$ & 4.39 & $<0.001$ & 3 & $2.08(0.43-6.09)$ & 0.96 & 0.2 \\
\hline
\end{tabular}

*Affer excluding acute leukemia

NA Blot applicable

4 


\section{Table 3(on next page)}

SIRs and excess risks after excluding secondary cancers which were diagnosed within first year after the diagnosis of CML

*Acute leukemia excluded Excess risk is per 10,000. Confidence intervals are $95 \%$. 
Table 3. SIRs and excess risks after excluding secondary cancers which were diagnosed within first year after the diagnosis of CML.

\begin{tabular}{|c|c|c|c|c|c|c|}
\hline Cancer sites & Observed & O/E & Cl Lower & CI Upper & $\begin{array}{l}\text { Excess } \\
\text { Risk }\end{array}$ & 'P' value \\
\hline $\begin{array}{l}\text { All Sites excluding Non-Melanoma } \\
\text { Skin* }\end{array}$ & 405 & 1.26 & 1.12 & 1.4 & 26.45 & $<0.001$ \\
\hline All Solid Tumors & 350 & 1.21 & 1.09 & 1.34 & 19.27 & $<0.001$ \\
\hline Oral Cavity and Pharynx & 12 & 1.41 & 0.73 & 2.46 & 1.11 & 0.23 \\
\hline Gum and Other Mouth & 4 & 3.6 & 1.3 & 9.22 & 0.92 & 0.006 \\
\hline Digestive System & 76 & 1.23 & 0.97 & 1.54 & 4.46 & 0.08 \\
\hline Stomach & 12 & 2.20 & 1.14 & 3.85 & 2.08 & 0.004 \\
\hline Small Intestine & 4 & 2.47 & 0.67 & 6.33 & 0.76 & 0.06 \\
\hline Colon & 31 & 1.41 & 0.96 & 2 & 2.85 & 0.83 \\
\hline Rectum & 4 & 0.61 & 0.16 & 1.55 & -0.83 & 0.32 \\
\hline Hepatobiliary System & 11 & 1.28 & 0.64 & 2.28 & 0.76 & 0.42 \\
\hline Liver & 9 & 1.56 & 0.71 & 2.96 & 1.02 & 0.18 \\
\hline Pancreas & 10 & 1.06 & 0.51 & 1.95 & 0.18 & 0.85 \\
\hline Respiratory System & 62 & 1.26 & 0.97 & 1.62 & 4.1 & 0.07 \\
\hline Lung and Bronchus & 60 & 1.31 & 1 & 1.69 & 4.53 & 0.03 \\
\hline Soft Tissue including Heart & 6 & 3.01 & 1.11 & 6.56 & 1.27 & 0.004 \\
\hline Skin excluding Basal and Squamous & 26 & 1.51 & 0.99 & 2.22 & 2.79 & 0.06 \\
\hline Melanoma of the Skin & 22 & 1.4 & 0.88 & 2.12 & 1.99 & 0.11 \\
\hline Female Breast & 23 & 0.63 & 0.4 & 0.94 & -4.35 & 0.03 \\
\hline Female Genital System & 22 & 1.54 & 0.97 & 2.33 & 2.45 & 0.07 \\
\hline Cervix Uteri & 4 & 2.53 & 0.69 & 6.47 & 0.77 & 0.06 \\
\hline Corpus Uteri & 9 & 1.19 & 0.54 & 2.25 & 0.45 & 0.67 \\
\hline Ovary & 5 & 1.41 & 0.46 & 3.29 & 0.46 & 0.44 \\
\hline Male Genital System & 76 & 1.29 & 1.02 & 1.62 & 5.48 & 0.03 \\
\hline Prostate & 75 & 1.31 & 1.03 & 1.64 & 5.59 & 0.02 \\
\hline Urinary System & 37 & 1.25 & 0.88 & 1.72 & 2.33 & 0.17 \\
\hline Urinary Bladder & 21 & 1.21 & 0.75 & 1.85 & 1.16 & 0.38 \\
\hline
\end{tabular}




\begin{tabular}{|lcccccc|}
\hline Kidney & 14 & 1.29 & 0.7 & 2.16 & 0.99 & 0.35 \\
\hline Endocrine System & 12 & 1.85 & 0.95 & 3.23 & 1.74 & 0.12 \\
\hline Thyroid & 12 & 1.95 & 1.01 & 3.41 & 1.86 & $\mathbf{0 . 0 2}$ \\
\hline Hematological malignancies * & 47 & 1.8 & 1.1 & 2.5 & 6.68 & $<0.001$ \\
\hline Non-Hodgkin Lymphoma & 17 & 1.22 & 0.71 & 1.95 & 0.97 & 0.42 \\
\hline Myeloma & 8 & 1.62 & 0.7 & 3.18 & 0.97 & 0.16 \\
\hline Chronic Lymphocytic Leukemia & 11 & 2.87 & 1.43 & 5.14 & 2.27 & $<0.001$ \\
\hline
\end{tabular}

*Acute leukemia excluded

Excess risk is per 10,000.

Confidence intervals are $95 \%$.

$$
\# \mathrm{P}<0.05
$$

